\title{
Renal Function Assessment Gap in Clinical Practice: An Awkward Truth
}

\author{
Francesco Trevisani ${ }^{a, b}$ Federico Di Marco ${ }^{b} \quad$ Umberto Capitanio ${ }^{a, b}$ \\ Alessandro Larcher ${ }^{a}$ b Arianna Bettiga $^{b}$ Flaviano Dosio ${ }^{c}$ \\ Michele Ghidinid $^{d} \quad$ Gianluca Del Conte $^{\mathrm{e}}$ Riccardo Vago $^{\mathrm{b}}$ \\ Alessandra Cinque ${ }^{b}$ Luigi Gianollic Andrea Salonia $a, b$ \\ Alberto Brigantia, b Sergio Luis-Lima $^{f} \quad$ Natalia Negrín-Mena ${ }^{f}$ \\ Francesco Montorsi ${ }^{a, b}$ Esteban Porrini ${ }^{f}$
}

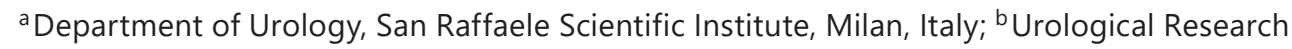
Institute, San Raffaele Scientific Institute, Milan, Italy; ' Nuclear Medicine Unit, Scientific Institute San Raffaele, Milan, Italy; ${ }^{\mathrm{d}}$ Medical Oncology Unit, Fondazione IRCCS Ca' Granda Ospedale Maggiore Policlinico, Milan, Italy; ${ }^{e}$ Department of Oncology, San Raffaele Scientific Institute, Milan, Italy; ${ }^{f}$ Department of Nephrology, University Hospital of the Canary Island, University of La Laguna, Tenerife, Spain

\section{Keywords}

Renal function - Serum creatinine $\cdot$ Cystatin C $\cdot$ Glomerular filtration rate $\cdot$ Renal measurement techniques - Estimated glomerular filtration rate $\cdot$ Measured glomerular filtration rate

\begin{abstract}
Introduction: An accurate assessment of renal function is needed in the majority of clinical settings. Unfortunately, the most used estimated glomerular filtration rate (eGFR) formulas are affected by significant errors in comparison to gold standards methods of measured GFR (mGFR). Objective: The objective of the study is to determine the extent of the error of eGFR formulas compared to the mGFR in different specific clinical settings. Methods: A total retrospectively consecutive cohort of 1,320 patients (pts) enrolled in 2 different European Hospitals (Center 1: 470 pts; Center 2: 850 pts) was collected in order to compare the most common eGFR formulas used by physicians with the most widespread mGFR methods in daily clinical practice (lohexol Plasma Clearance -Center 1 [mGFR-iox] and Renal Scintigraphy -Center 2 [mGFR-scnt]). The study cohort was composed by urological, oncological, and nephrological pts. The agreement between eGFR and mGFR was evaluated using bias (as median of difference), precision (as interquartile range of difference) accuracy (as $\mathrm{P}_{30}$ ), and total deviation index. Results: The most accurate eGFR formula in the comparison with gold standard method (lohexol plasma clearance) in Center 1 was represented by s-creatinine and cystatin C combined Chronic Kidney Disease-Epidemiology Collaboration-cr-cy, even though the $\mathrm{P}_{30}$ is re-
\end{abstract}




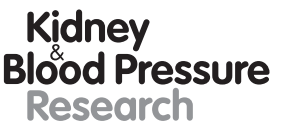

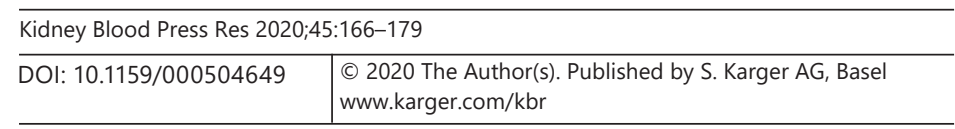

Trevisani et al.: New Challenges in Renal Function Assessment

duced (84\%) under the threshold of $60 \mathrm{~mL} / \mathrm{min} / 1.73 \mathrm{~m}^{2}$. Similar results were found in Center 2, with a wider discrepancy between mGFR-scnt and eGFR formulas due to the minor accuracy of the nuclear tool in respect to the mGFR-iox. Conclusions: The loss of accuracy observed for the formulas at lower values of GFR suggests the mandatory use of gold standards methods as lohexol Plasma Clearance to assess the correct status of renal function for critical cases. The center 2 showed lower levels of agreement between mGFR and eGFR suggesting that the errors are partially accounted for the Renal Scintigraphy technique too. In particular, we suggest the use of mGFR-iox in oncological urological and nephrological pts with an eGFR lower than $60 \mathrm{~mL} / \mathrm{min} / 1.73 \mathrm{~m}^{2}$.

(C) 2020 The Author(s)

Published by S. Karger AG, Basel

\section{Introduction}

Glomerular filtration rate (GFR) can be assessed by 2 different categories of methods, the estimated ones and the measured ones. The former group aims to indirectly estimate GFR (eGFR) using formulas based on endogenous markers such as creatinine and/or cystatin-C. The latter one intends to measure straight the GFR (mGFR) by means of the pharmacokinetic analysis of exogenous substances, such as inulin, radio-labeled markers $\left({ }^{51} \mathrm{Cr}\right.$-EDTA, ${ }^{99 \mathrm{~m} T c-}$ DTPA), or nonlabeled contrast media (iohexol, iothalamate) [1, 2].

Indirect methods are fast, user-friendly, and cost-effective while direct ones deserve time, skills, and a specific equipment $[3,4]$.

Several papers in literature have already demonstrated that GFR calculated by formulas shows an average error in reflecting the real GFR of about $\pm 30 \%$ in patients (pts) affected by type 2 diabetes, chronic kidney diseases, autosomal polycystic kidney diseases, and in renal transplantation [5]. Surprisingly, there is a scarce evidence on the error of eGFR in pts with urological and oncological diseases, even though an accurate assessment of renal function is of paramount importance in that specific clinical setting [6].

As a matter of fact, in renal cancer pts, preoperative kidney function represents one of the major factors affecting relevant decision making like selecting conservative versus radical surgery [7]. Similarly, the dosage of several oncological nephrotoxic therapies is based on eGFR or creatinine clearance and not on mGFR in several metastatic urological malignancies [8]. Moreover, the most prominent clinical trials of the new anticoagulants agents are still based on Cockroft Gault formula [9].

Finally, a precise evaluation of GFR is crucial in the evaluation of possible living kidney donors and in kidney recipients after transplantation [5, 10].

In this study, we analyzed the agreement between mGFR and eGFR in the evaluation of renal function in 2 different Centers with different clinical settings, with a particular focus on the oncological and urological pts.

\section{Materials and Methods}

Population Study

This is a cross-sectional retrospective study involving 2 different hospitals: Hospital Universitario de Canarias (Tenerife-Spain; Center 1) and San Raffaele Hospital (Milano-Italy; Center 2). These 2 centers perform mGFR on a routine basis to pts with diverse clinical conditions. Inclusion criteria were (a) prostate, bladder, kidney, and testis urological cancer, (b) nonurological cancer with urological involvement (e.g., hydronephrosis) for whom is necessary to estimate the renal function to use the correct dose of immunotherapy treatment 


\section{Kidney \\ Blood Pressure \\ Research}

Table 1. Clinical characteristics of the study cohorts

\begin{tabular}{l|l}
\hline Kidney Blood Press Res 2020;45:166-179 \\
\hline DOI: 10.1159/000504649 & $\begin{array}{l}\text { @ 2020 The Author(s). Published by S. Karger AG, Basel } \\
\text { www.karger.com/kbr }\end{array}$ \\
\hline
\end{tabular}

\begin{tabular}{lc}
\hline Center 1 & \\
Number & 470 \\
Age, years, median (IQR) & $57(48-78)$ \\
Gender, male, $n(\%)$ & $305(65)$ \\
Nephrological disease, $n(\%)$ & $252(54)$ \\
Kidney donor, $n(\%)$ & $86(18)$ \\
Kidney transplant, $n(\%)$ & $252(20)$ \\
mGFR, mL/min/1.73 m ${ }^{2,}$ mean, (SD) & $47.9(27.8)$ \\
Serum creatinine, mg/dL, mean, (SD) & $1.92(1.25)$ \\
Serum cystatin C, g/dL, mean, (SD) & $1.91(0.98)$ \\
\hline Center 2 & \\
Number & 850 \\
Age, years, median (IQR) & $61(45-72)$ \\
Gender, male, $n(\%)$ & $498(59)$ \\
Nephrological disease, $n(\%)$ & $124(15)$ \\
Urological functional disease, $n$ (\%) & $396(47)$ \\
Oncological, $n(\%)$ & $282(33)$ \\
Kidney donor, $n(\%)$ & $48(5)$ \\
mGFR, mL/min/1.73 m ${ }^{2,}$ mean, (SD) & $75.7(32.8)$ \\
Serum creatinine, mg/dL, mean, (SD) & $1.22(0.67)$ \\
\hline \multicolumn{2}{c}{ IQR, interquartile range; mGFR, measured glomerular filtration } \\
rate.
\end{tabular}

(breast, colorectal, lung, uterus, ovary, and others), (c) benign urological diseases (pelviureteric junction obstruction, stones, ureteral stenosis, hydronephrosis, endometriosis, and neurological bladder), (d) living kidney donors, (e) renal transplanted pts, and (f) pts with nephrological diseases with different grades of chronic kidney disease according the KDIGO guidelines. Exclusion criteria include (a) age $<18$ years, (b) genetic diseases such as Von Hippel Lindau and acquired autosomal polycystic kidney disease hereditary. The clinical and pathological features of pts are resumed in Table 1.

\section{Measured Glomerular Filtration Rate}

Renal function was measured with the following methods:

Plasma clearance of iohexol (mGFR-iox; Center $1-n=470$ ). In the morning of examination, $5 \mathrm{~mL}$ of iohexol (Omnipaque 300, GE-Healthcare) was injected intravenously during 2 min [11]. Afterward, venous or capillary blood was obtained by finger prick at 120, 180, $240,300,360,420$, and $480 \mathrm{~min}$ for pts with eGFR $\leq 40 \mathrm{~mL} / \mathrm{min} / 1.73 \mathrm{~m}^{2}$; or at $120,150,180$, 210 , and $240 \mathrm{~min}$ for those with eGFR $>40 \mathrm{~mL} / \mathrm{min} / 1.73 \mathrm{~m}^{2}$. Iohexol was measured in plasma or dried blood spot (DBS) as previously shown [12]. Both methods using plasma or DBS showed excellent agreement and can be considered interchangeable [12]. Iohexol levels were measured by HPLC, as previously described. For the DBS analysis, a fixed volume of capillary blood $(10 \mu \mathrm{L})$ was taken by a capillary pipette and deposited on filter paper [12]. Finally, a circle of filter paper containing the whole drop of blood was punched out for analysis [12]. Plasma iohexol clearance was calculated according to a one-compartment model and then corrected by the formula proposed by Bröchner-Mortensen [11].

Renal scintigraphy using Tc 99m-DTPA (mGFR-scnt; Center $2-n=850$ ). Renal scans were performed according to published guidelines [13], and all subjects were instructed to drink at least $500 \mathrm{~mL}$ of water in the $30 \mathrm{~min}$ before examination. After i.v. injection on Tc 99m-DTPA (111-185 MBq), a kidney posterior view was obtained for $30 \mathrm{~min}$ in supine position using a gamma camera (Infinia, General Electric Healthcare) equipped with a LEHR collimator and a 


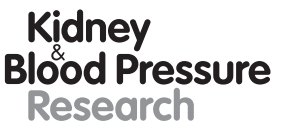

Research

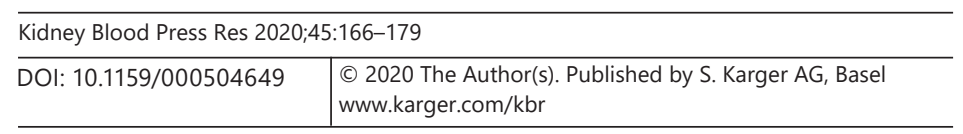

Trevisani et al.: New Challenges in Renal Function Assessment

dedicated computer to store all images. Each renal scan was processed using Gates method to analyze the mGFR-scnt, taking into consideration height, weight, and body surface to compute mGFR-scnt, expressed as $\mathrm{mL} / \mathrm{min} / 1.73 / \mathrm{m}^{2}$ of body surface.

\section{Estimated GFR by Formulas}

Simultaneously to the measurement of GFR, serum creatinine alone (Center 2) or together with cystatin-C (Center 1 ) were collected to calculate commonly used equations creatininebased: Chronic Kidney Disease-Epidemiology Collaboration (CKD-EPI-cr) [14], Modification of Diet in Renal Disease (MDRD) [15], Full Age Spectrum (FAS) [16], Mayo Clinic Quadratic (MCQ) [17], cystatin-C based: Le Bricon [18], Rule [19] and CKD-EPI-cy [20], and creatinine-cystatin-C based: CKD-EPI-cr-cy [20]. Formulas equations are reported in online supplementary Table 1 (see www.karger.com/doi/10.1159/000504649 for all online suppl. material).

The agreement between formulas and mGFR was evaluated with values adjusted for body surface area.

\section{Biochemistry}

Creatinine was measured by Jaffè IDMS-traceable creatinine (cobas c711 module, Roche Diagnostics; Center 1) or Kinetic Picrate (COBAS C 800) IMDS standardized (Center 2) and cystatin-C levels by immunonephelometry (the BN II System, Siemens Healthcare Diagnostics), calibrated with ERM-DA471/IFCC (Center 2).

\section{Statistical Analysis}

The performance of eGFR in reflecting mGFR was assessed by statistics of agreement evaluating 3 parameters usually used for these comparisons [21]: bias, precision, and overall accuracy. Bias is expressed as the median of the percent difference mGFR-eGFR and should indicate if the eGFR harbors systematic errors among the population. Precision is expressed as interquartile range (IQR) of the difference mGFR-eGFR and represents the variability of the difference among the average difference. The overall accuracy is evaluated using the $\mathrm{P}_{30}$ parameters which indicate the percent of estimates within $30 \%$ of mGFR. The agreement was also evaluated using percentage total deviation index (TDI) as metric [22]: the TDI calculates a percentage value such that the $95 \%$ of the percentages differences between measurements and estimations will be lower than this. Finally, the percentage of pts whose eGFR differs from the mGFR for $>5,10,15$, and $20 \mathrm{~mL} / \mathrm{min} / 1.73 \mathrm{~m}^{2}$ was evaluated in the cases with mGFR $\leq 60$ $\mathrm{mL} / \mathrm{min} / 1.73 \mathrm{~m}^{2}$. The analysis was performed using R-Studio environment for R version 3.6.0.

\section{Results}

The 2 cohorts of pts were treated separately.

\section{Center 1}

In the Center 1, GFR was measured using Iohexol Plasma Clearance. The comparisons between the mGFR-iox and the 8 considered formulas showed that some of the estimated methods are biased for the comparisons in our cohort. In particular, CKD-EPI-cr, MCQ, FAS, and Le Bricon tend to overestimate the GFR with a median of, respectively, 6, 25, 12, and 25\%; Rule equation tends to underestimate with a median of 12\%. MDRD, CKD-EPI-cy, and CKDEPI-cr-cy seem to be the unbiased methods in our cohort with a median difference of 0,2 (underestimation), and $0 \%$. Evaluating the precision of the formulas, all the IQRs are included between 11 and $14 \mathrm{~mL} / \mathrm{min} / 1.73 \mathrm{~m}^{2}$, except for the MCQ with $32 \mathrm{~mL} / \mathrm{min} / 1.73 \mathrm{~m}^{2}$. The $\mathrm{P}_{30}$ 


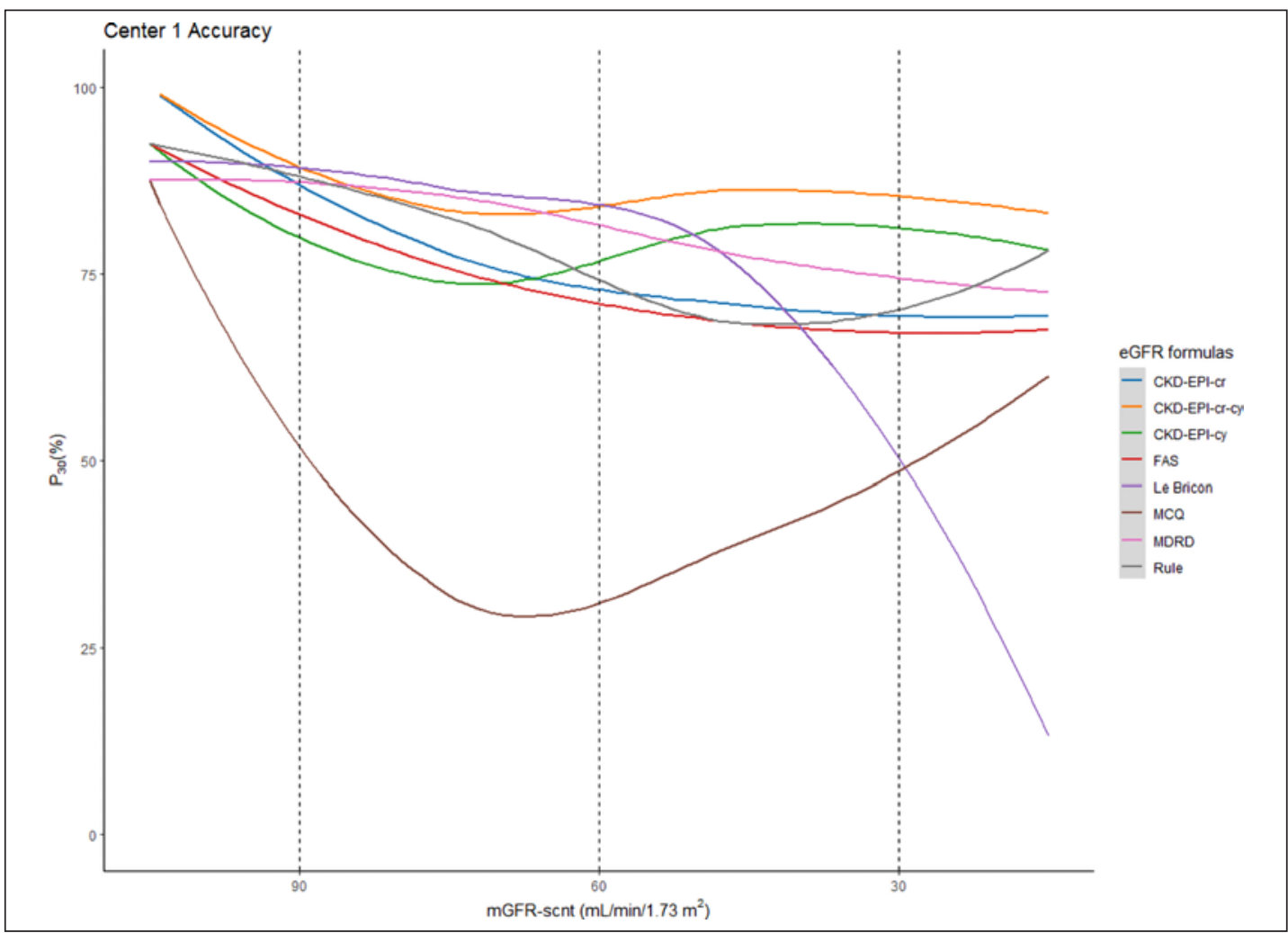

Fig. 1. Accuracy trend of EGFR formulas compared different MGFR intervals in center 1. eGFR, estimated glomerular filtration rate; mGFR, measured glomerular filtration rate; CKD-EPI, Chronic Kidney DiseaseEpidemiology Collaboration; MCQ, Mayo Clinic Quadratic.

Table 2. Accuracy, bias and precision for eGFR formula compared to mGFR-iox in center 1

\begin{tabular}{llrlr}
\hline Formula & $\mathrm{P}_{30, \%}$ & Bias, $\%$ & $\begin{array}{l}\text { IQR, mL/min/ } \\
1.73 \mathrm{~m}^{2}\end{array}$ & TDI, \% \\
\hline CKD-EPI-cr & 74 & -6 & 14 & 70.6 \\
MDRD & 78 & 0 & 12 & 65.3 \\
MCQ & 49 & -25 & 32 & 117 \\
FAS & 72 & -12 & 14 & 74.1 \\
Rule & 77 & 13 & 11 & 51.8 \\
CKD-EPI-cy & 80 & 2 & 12 & 50.9 \\
Le Bricon & 58 & -25 & 11 & 99.5 \\
CKD-EPI-cr-cy & 85 & 0 & 11 & 43.9 \\
\hline
\end{tabular}

IQR, interquartile range; eGFR, estimated glomerular filtration rate; mGFR, measured glomerular filtration rate; CKD-EPI, Chronic Kidney Disease-Epidemiology Collaboration; MCQ, Mayo Clinic Quadratic; TDI, total deviation index.

showed the accuracy for all the formulas: in this cohort, the CKD-EPI-cr-cy resulted in the most accurate with a value of $85 \%$, whereas the MCQ and Le Bricon resulted in the less accurated with, respectively, 49 and 58\%. All the other formulas were included between 72 and $80 \%$. The TDI calculation reported similar results confirming the highest agreement for CKDEPI-cr-cy (43.9\%) and low agreement for MCQ (117\%) and Le Bricon (99.5\%; Table 2). 
Table 3. Accuracy P30 for formulas versus mGFR-iox in cohort 1 stratified by CKD stages

\begin{tabular}{lllllllll}
\hline CKD stage & \multicolumn{1}{l}{$\mathrm{P}_{30}, \%$} & & & & \\
\cline { 2 - 8 } & CKD-EPI-cr & MDRD & MCQ & FAS & CKD-EPI-cy & rule & lebricon & CKD-EPI-cr-cy \\
\hline I & 100 & 88 & 88 & 93 & 93 & 93 & 90 & 100 \\
II & 78 & 85 & 32 & 76 & 74 & 83 & 86 & 83 \\
III & 71 & 77 & 40 & 68 & 81 & 68 & 75 & 86 \\
IV & 69 & 73 & 61 & 68 & 78 & 78 & 13 & 83 \\
\hline
\end{tabular}

mGFR, measured glomerular filtration rate; CKD-EPI, Chronic Kidney Disease-Epidemiology Collaboration; MCQ, Mayo Clinic Quadratic.

Table 4. Percentages of patients with mGFR-iox lower than $60 \mathrm{~mL} / \mathrm{min} / 1.73 \mathrm{~m}^{2}$ whom eGFR differs from mGFR-iox at different thresholds in center 1

\begin{tabular}{llll}
\hline & $\begin{array}{l}\text { Differences up to } \\
5 \mathrm{~mL} / \mathrm{min} / 1.73 \mathrm{~m}^{2, \%}\end{array}$ & $\begin{array}{l}\text { Differences from } 5 \text { to } \\
20 \mathrm{~mL} / \mathrm{min} / 1.73 \mathrm{~m}^{2, \%}\end{array}$ & $\begin{array}{l}\text { Differences over } 20 \mathrm{~mL} / \\
\mathrm{min} / 1.73 \mathrm{~m}^{2, \%}\end{array}$ \\
\hline CKD-EPI-cr & 50 & 41 & 9 \\
MDRD & 56 & 37 & 7 \\
MCQ & 39 & 33 & 28 \\
FAS & 55 & 34 & 11 \\
Rule & 49 & 47 & 4 \\
CKD-EPI-cy & 54 & 44 & 2 \\
Le Bricon & 21 & 74 & 5 \\
CKD-EPI-cr-cy & 59 & 38 & 3 \\
\hline
\end{tabular}

eGFR, estimated glomerular filtration rate; mGFR, measured glomerular filtration rate; CKD-EPI, Chronic Kidney Disease-Epidemiology Collaboration; MCQ, Mayo Clinic Quadratic.

The $\mathrm{P}_{30}$ was also evaluated at different range of GFR, observing an overall high accuracy for high values of GFR (higher than $90 \mathrm{~mL} / \mathrm{min} / 1.73 \mathrm{~m}^{2}$ ) with the CKD-EPI-cr and CKD-EPIcr-cy scoring a 100\% and the others between 88 and $93 \%$. For lower values of GFR, a common loss of accuracy was observed with the Le Bricon formulas showing a value of $13 \%$ at GFR under $30 \mathrm{~mL} / \mathrm{min} / 1.73 \mathrm{~m}^{2}$ (Fig. 1).

The accuracy of the eGFR formulas was evaluated for each stage of CKD according to mGFR-iox (Table 3), observing a decay of $\mathrm{P}_{30}$ at lower stages.

Considering the pts with mGFR-iox lower than $60 \mathrm{~mL} / \mathrm{min} / 1.73 \mathrm{~m}^{2}$, we calculated the percentages of pts who had an estimated value that differed from measurement by $<5 \mathrm{~mL} /$ $\min / 1.73 \mathrm{~m}^{2}$, between $5 \mathrm{~mL} / \mathrm{min} / 1.73 \mathrm{~m}^{2}$ and $20 \mathrm{~mL} / \mathrm{min} / 1.73 \mathrm{~m}^{2}$ and over $20 \mathrm{~mL} / \mathrm{min} / 1.73$ $\mathrm{m}^{2}$ for all the formulas (Table 4).

In particular, we observed that the eGFR, calculated with the most common formulas based on serum creatinine and/or serum cystatin C, demonstrated in the $52 \%$ of pts a discrepancy higher than $5 \mathrm{~mL} / \mathrm{min} 1.73 \mathrm{~m}^{2}$ in comparison to the gold standard.

Accuracy analysis based on age tertiles is presented in online supplementary Table 2 for both cohorts

Center 2

Different results were obtained from the Center 2 cohort where renal scintigraphy was adopted to measure the GFR. In this case only, the 4 creatinine-based formulas were used, and 


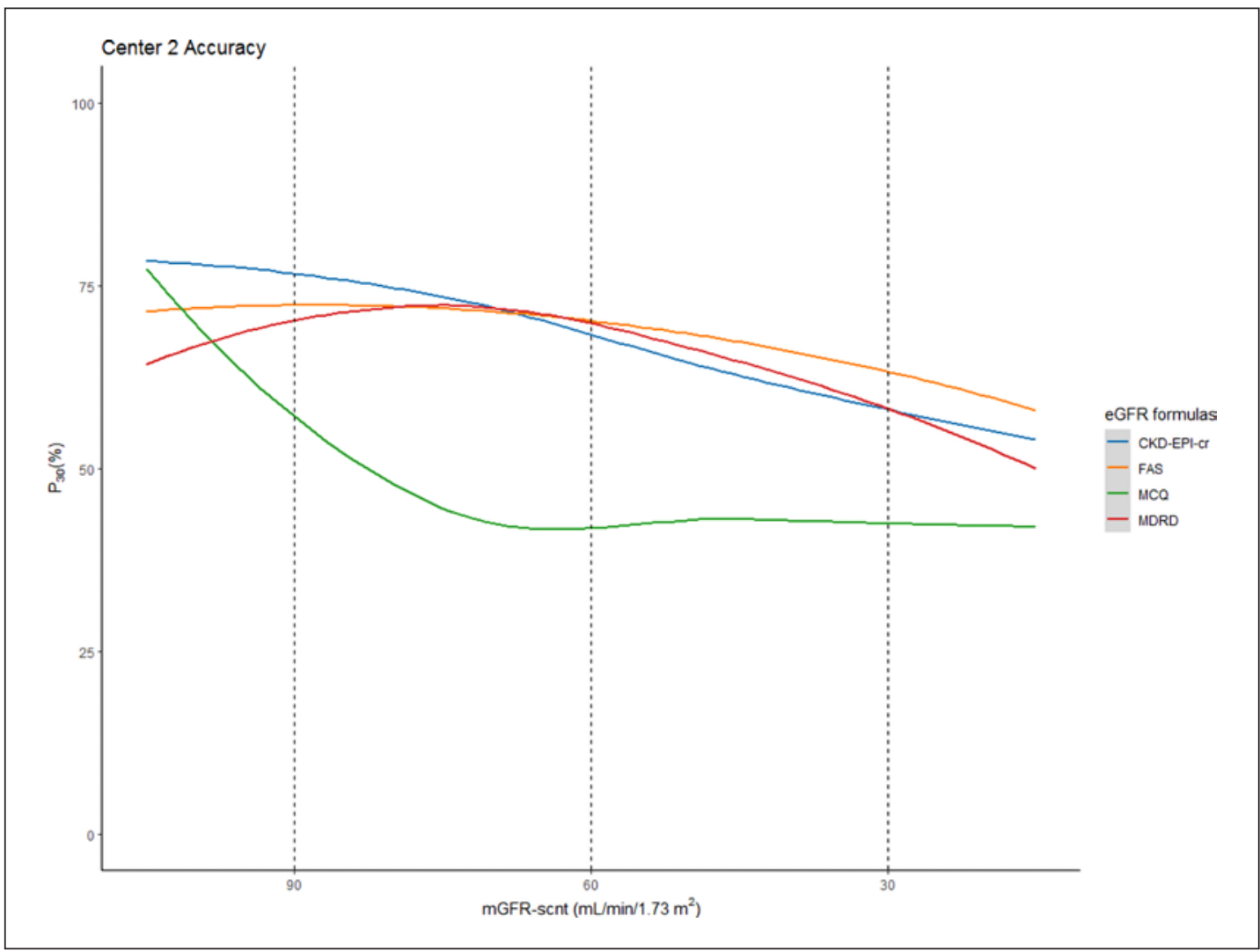

Fig. 2. Accuracy trend of EGFR formulas compared to different MGFR intervals in center 2. eGFR, estimated glomerular filtration rate; mGFR, measured glomerular filtration rate; CKD-EPI, Chronic Kidney DiseaseEpidemiology Collaboration; MCQ, Mayo Clinic Quadratic.

we observed that the formulas tend to over/underestimate the mGFR-scnt value as follows: CKD-EPI-cr, MDRD, and FAS median underestimation of 6, 10, and 7\% while MCQ overestimation of $16 \%$. The IQR resulted higher for Center 2 with values between 25 and $35 \mathrm{~mL} /$ $\min / 1.73 \mathrm{~m}^{2}$. The overall accuracy resulted lower observing $71 \%$ for CKD-EPI-cr, $66 \%$ for MDRD, 70\% for FAS, and 55\% for MCQ (higher than Center 1). The TDI remained in line with Center 1 confirming the low agreement for MCQ formula (97.6\%; Table 5).

As for Center 1, the accuracy at different ranges showed a loss of accuracy at lower values of GFR (Fig. 2) and lower CKD stages (Table 6).

Considering the pts with mGFR-scnt lower than $60 \mathrm{~mL} / \mathrm{min} / 1.73 \mathrm{~m}^{2}$, we observed that also in this cohort, for MCQ the percentages of pts with a difference of $20 \mathrm{~mL} / \mathrm{min} / 1.73 \mathrm{~m}^{2}$ or higher is the biggest among all formulas with a value of $39 \%$ against the mean $15 \%$ of the others. For differences lower than $5 \mathrm{~mL} / \mathrm{min} / 1.73 \mathrm{~m}^{2}$, the mean was $24.5 \%$, while between 5 and $20 \mathrm{~mL} / \mathrm{min} / 1.73 \mathrm{~m}^{2}$ was $54.5 \%$ with all the percentages being comparable between formulas (Table 7).

\section{Discussion}

Intro

In daily clinical practice, the use of an "ideal" marker of renal damage uncorrelated to serum creatinine such as inulin, iothalamate, diethylene, triamine penta-acetic acid, or iohexol 
Table 5. Accuracy, bias, and precision for eGFR formula compared to mGFR-scnt in center 2
Table 6. Accuracy P30 for formulas versus mGFR-scnt in cohort 2 stratified by CKD stages

\begin{tabular}{llrll}
\hline Formula & $\mathrm{P}_{30, \%}$ & Bias, \% & $\begin{array}{l}\mathrm{IQR}, \mathrm{mL} / \mathrm{min} / \\
1.73 \mathrm{~m}^{2}\end{array}$ & $\mathrm{TDI}, \%$ \\
\hline CKD-EPI-cr & 71 & 6 & 26 & 65.9 \\
MDRD & 66 & 10 & 29 & 68.7 \\
MCQ & 55 & -16 & 35 & 97.6 \\
FAS & 70 & 7 & 25 & 67.9 \\
\hline
\end{tabular}

eGFR, estimated glomerular filtration rate; mGFR, measured glomerular filtration rate; CKD-EPI, Chronic Kidney Disease-Epidemiology Collaboration; MCQ, Mayo Clinic Quadratic; IQR, interquartile range; TDI, total deviation index.

\begin{tabular}{lllll}
\hline \multirow{2}{*}{ CKD stage } & \multicolumn{2}{l}{$\mathbf{P}_{30,} \%$} & & \\
\cline { 2 - 5 } & CKD-EPI-cr & MDRD & MCQ & FAS \\
\hline I & 78 & 64 & 77 & 71 \\
II & 73 & 72 & 45 & 72 \\
III & 63 & 65 & 43 & 67 \\
IV & 54 & 50 & 42 & 58 \\
\hline
\end{tabular}

CKD, chronic kidney disease; mGFR, measured glomerular filtration rate; CKD-EPI, CKD-Epidemiology Collaboration; MCQ, Mayo Clinic Quadratic.

Table 7. Percentages of patients with mGFR-scnt lower than $60 \mathrm{~mL} / \mathrm{min} / 1.73 \mathrm{~m}^{2}$ whom eGFR differs from mGFR-scnt at different thresholds in center 2

\begin{tabular}{llll}
\hline & $\begin{array}{l}\text { Differences up to } \\
5 \mathrm{~mL} / \mathrm{min} / 1.73 \mathrm{~m}^{2, \%}\end{array}$ & $\begin{array}{l}\text { Differences from } 5 \text { to } \\
20 \mathrm{~mL} / \mathrm{min} / 1.73 \mathrm{~m}^{2, \%}\end{array}$ & $\begin{array}{l}\text { Differences over } \\
20 \mathrm{~mL} / \mathrm{min} / 1.73 \mathrm{~m}^{2, \%}\end{array}$ \\
\hline CKD-EPI-cr & 24 & 58 & 18 \\
MDRD & 30 & 58 & 12 \\
MCQ & 16 & 45 & 39 \\
FAS & 28 & 57 & 15 \\
\hline
\end{tabular}

eGFR, estimated glomerular filtration rate; mGFR, measured glomerular filtration rate; CKD-EPI, Chronic Kidney Disease-Epidemiology Collaboration; MCQ, Mayo Clinic Quadratic.

is often discouraged because expensive and time consuming [4]. For these reasons, different types of formulas for estimating GFR have been proposed, driving nowadays clinical decisions in many hospitals and laboratories [15].

\section{Center 1}

The Center 1 is a nephrological department where most of the pts suffered from nephrological diseases or end-stage renal diseases treated with kidney transplant. In general, the $\mathrm{P}_{30}$ for all the equations evaluated averaged $72 \%$ ranging from 50 to $85 \%$. This means that 50 to $85 \%$ of the estimations had an error up to $30 \%$ from mGFR-iox. Importantly, this error was 


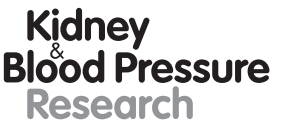

Kidney Research

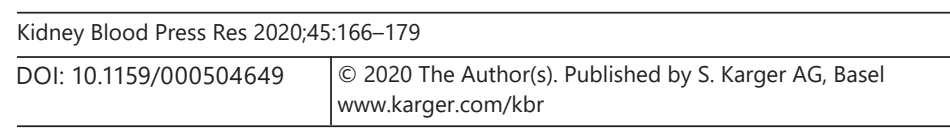

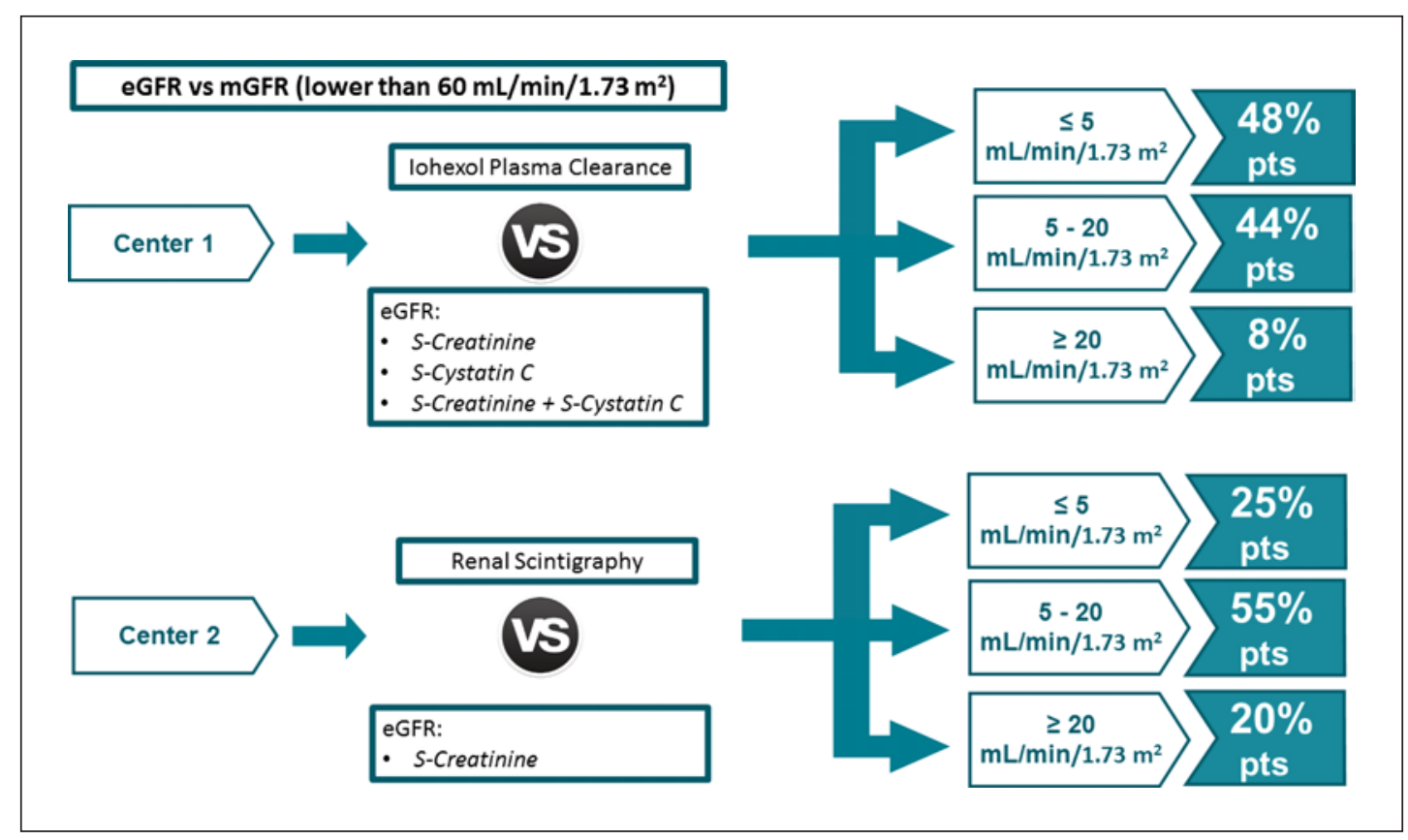

Fig. 3. Mean difference at different thresholds for pts with MGFR lower than $60 \mathrm{~mL} / \mathrm{min} / 1.73 \mathrm{~m}^{2}$. eGFR, estimated glomerular filtration rate; mGFR, measured glomerular filtration rate.

larger than $30 \%$ in a nonneglectable number of cases: 50 or $40 \%$ for the Le Bricon and MCQ equations. Thus, large variability between eGFR and mGFR-iox is frequent and severe. The agreement, expressed as TDI, confirmed this situation, showing that, to obtain the $95 \%$ of the estimates, a boundary is needed of at least $44 \%$ in the best-case scenario (CKD-EPI-cr-cy).

It is important to notice that a systematic error (bias) occurs for 5 of the 8 formulas considered, while CKD-EPI-cr-cy, MDRD, and CKD-EPI-cy turned out to have a negligible bias.

The comparisons between the eGFR formulas and mGFR-iox show that there is a loss of accuracy at lower values of GFR $\left(\leq 60 \mathrm{~mL} / \mathrm{min} / 1.73 \mathrm{~m}^{2}\right)$ where the metrics $\mathrm{P}_{30}$ for all the formulas is lower than $85 \%$. In particular, a not negligible percentage of patient with mGFR-iox lower than $60 \mathrm{~mL} / \mathrm{min} / 1.73 \mathrm{~m}^{2}$, tends to be wrongly over or underestimated with a discrepancy $>20 \mathrm{~mL} / \mathrm{min} / 1.73 \mathrm{~m}^{2}$ (especially the MCQ formulas).

Surely the MCQ and Le Bricon formulas turned out to be the less effective for the GFR estimation with low accuracy ( 49 and 58\%) and the presence of a remarkable bias.

\section{Center 2}

The Center 2 is a urological/oncological department where the use of Renal Scintigraphy is mostly used to decide the best surgical/clinical approach as daily routine. As a matter of fact, even though iohexol technique represents the most accurate method to define the "real renal function," renal scintigraphy with TC 99 m-DTPA or MAG3 remains the most popular functional exam which urologists and oncologists prescribe to pts in clinical practice, thanks to the ability to determinate the separate function of kidneys. Our observations confirm the lower efficacy of the Renal Scintigraphy in the correct determination of GFR, showing a divergent scenario with the results obtained in Center 1 . In fact, while the accuracy of the eGFR on mGFR-iox is about 71\%, in the Center 2, mGFR-scnt decreases down to $65 \%$. Moreover, a systematic difference seems to be present for all the formulas referring to mGFRscnt and also the midspread of the difference is double than Center 1. 
Fig. 4. Distribution of the absolute differences between MGFR and EGFR in the 2 centers.

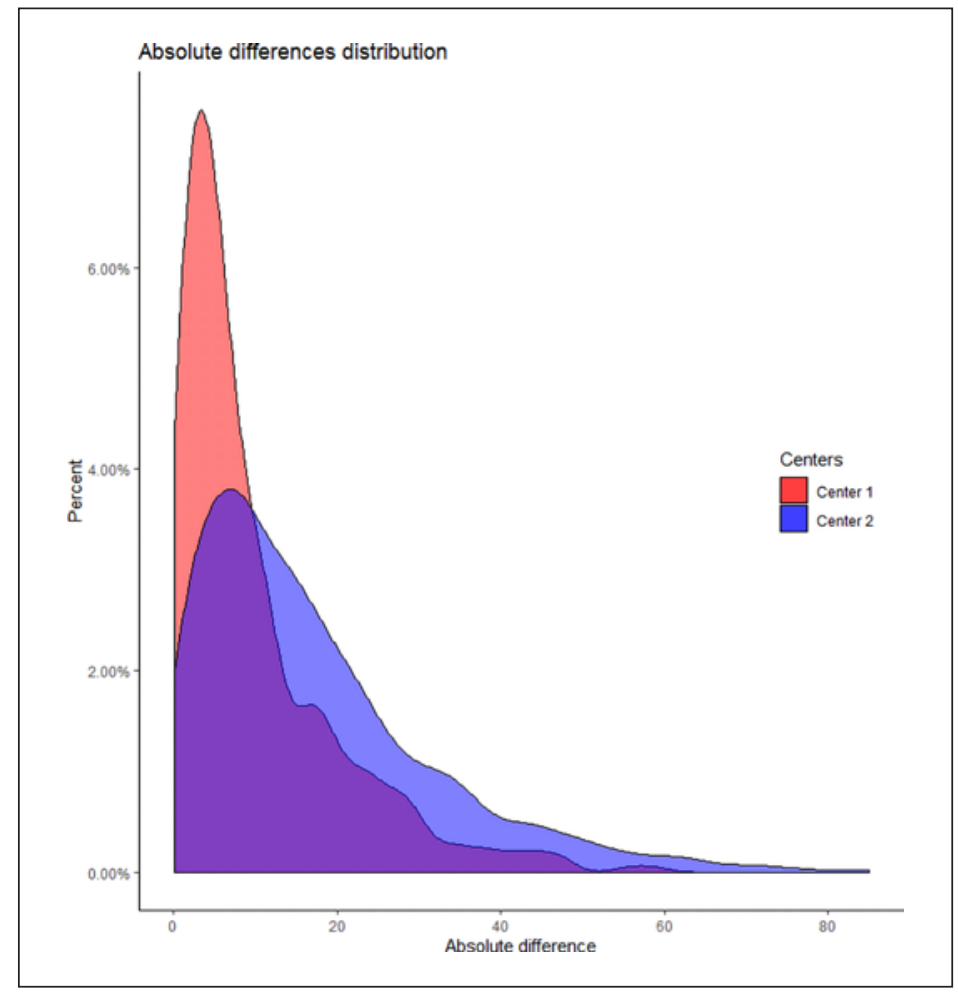

\section{Overall Considerations}

The analysis of our work has obviously elucidated 2 different but cumbersome points; the low reliability of eGFR formulas in comparison to the gold standard mGFR-iox method and the evident disagreement between eGFR and renal scintigraphy.

First (as shown in Fig. 3), the eGFR formulas in comparison to gold standard mGFR-iox appear less accurate to define renal function under $60 \mathrm{~mL} / \mathrm{min} / 1.73 \mathrm{~m}^{2}$, with $>50 \%$ of pts who are over or underestimated by $>5 \mathrm{~mL} / \mathrm{min} / 1.73 \mathrm{~m}^{2}$. Regarding the differences of iohexol renal measurement and renal scintigraphy in respect to eGFR, our work clearly demonstrates that the distribution of the absolute differences between mGFR and eGFR varies among the 2 techniques, with a well-spreaded trend in Center 2 and a narrower one in Center 1 (Fig. 4, 5). Nevertheless, renal scintigraphy remains the unique available technique able to define the separate renal function GFR, a key factor in the surgical management of kidney cancer or transplantation. For this argument, even though lower accuracy in comparison to iohexol plasma clearance, renal scintigraphy remains a valuable tool in clinical field. The 3 methods considered in this study, eGFR mGFR-iox, and mGFRscnt, and their features (respectively, ease of use, accuracy, and assessment of separate renal GFR) must be considered when approaching a patient during its therapeutic pathway (Fig. 6).

\section{Clinical Implications}

In this study, we considered 3 type of medical field in which assessment of GFR plays a key role: urological, oncological, and nephrological.

Regarding urological pts, the determination of the real renal function remains one of the cornerstones in daily clinical management. For instance, preoperative GFR is one of the most important variables which are considered during surgical planning (radical vs. partial 


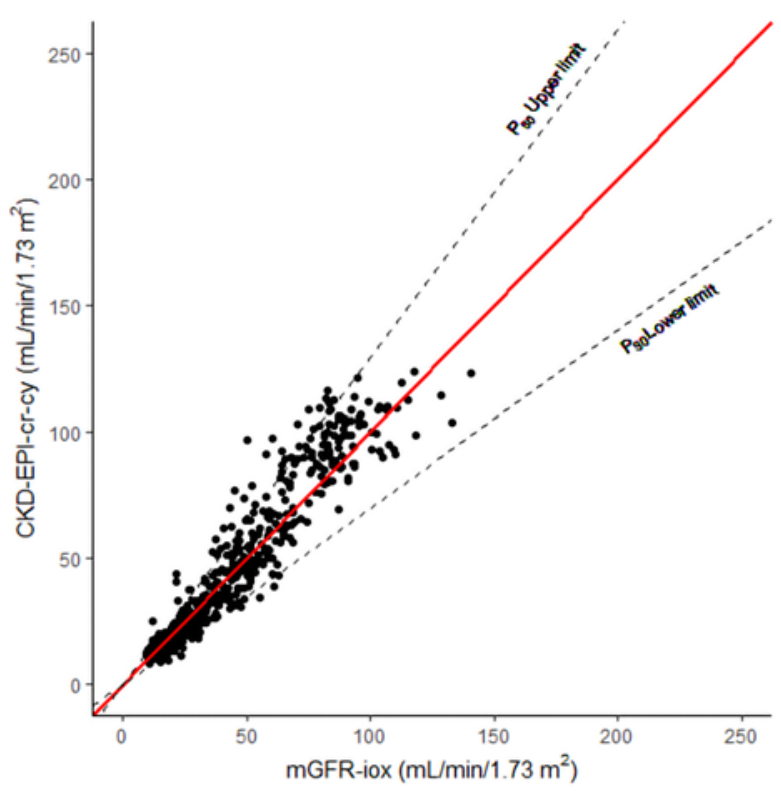

a

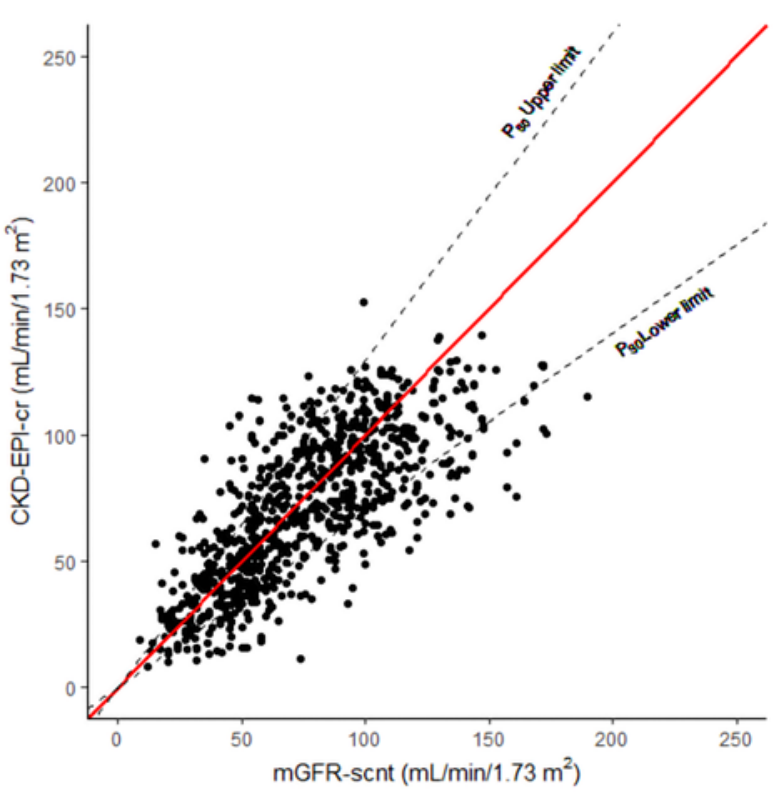

b

Fig. 5. Scatterplot of EGFR versus MGFR: (A) CKD-EPI-CR-CY versus MGFR-IOX; (B) CKD-EPI-CR versus MGFR-SCNT. Red lines represent identity and black dotted lines represent $\mathrm{P}_{30}$ boundaries. mGFR, measured glomerular filtration rate; CKD-EPI, Chronic Kidney Disease-Epidemiology Collaboration.

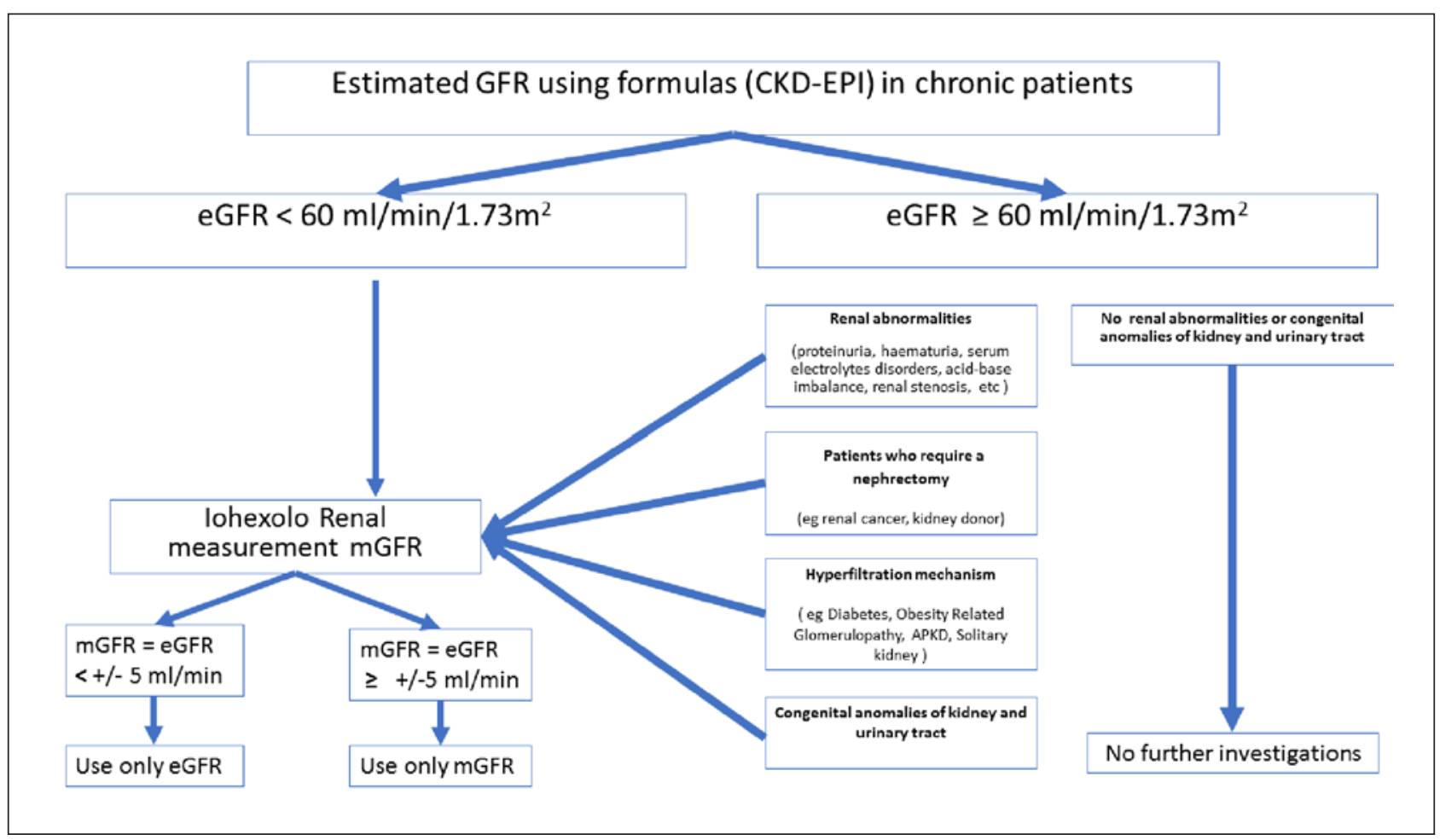

Fig. 6. Summarize of the decisional algorthm between MGFR and EGFR for chronic pts. eGFR, estimated glomerular filtration rate; mGFR, measured glomerular filtration rate; CKD-EPI, Chronic Kidney Disease-Epidemiology Collaboration. 


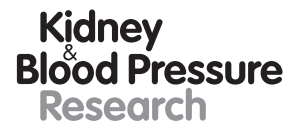

Kidney
Blood Pressure
Research

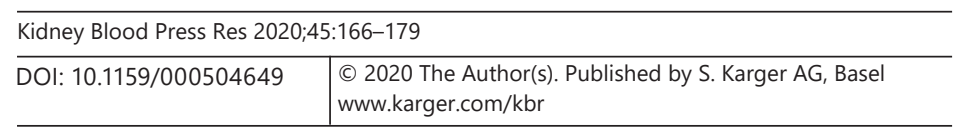

Trevisani et al.: New Challenges in Renal Function Assessment

nephrectomy) in kidney cancer pts [23]. Similarly, in kidney stones and pyelouretheral junction stenosis, split renal function evaluation is of paramount importance to decide if and how to operate [24]. For those and other aspects related both to research and clinical practice, GFR determination harbors a pivotal role for urologists.

Regarding oncological pts, the use of a reliable method to evaluate renal function is crucial since the prevalence of renal dysfunction (CKD and acute kidney injury) raised up in the last decade up to $50 \%$ as reported in some series [25]. The IRMA I and II studies, which included 5,000 adult cancer pts each, showed a rate of GFR lower than $90 \mathrm{~mL} / \mathrm{min} / 1.73 \mathrm{~m}^{2}$, respectively, in 52.9 and $50.2 \%$ of cases and a proportion of 12 and $11.8 \%$ of CKD stages III and IV [26]. In the IRMA-2 study, pts with a GFR lower than $60 \mathrm{~mL} / \mathrm{min} / 1.73 \mathrm{~m}^{2}$ had a lower mean survival compared to pts with better renal function (16.4 vs. 25.0 months for GFR superior to $60 \mathrm{~mL} / \mathrm{min} / 1.73 \mathrm{~m}^{2}$ ) in a population of cancer pts nonselected for type and stage of the tumor [27]. Furthermore, in CKD pts stage IIIa-b and IV, dose adjustment for chemotherapy is required to avoid nephrotoxicity and side effects that lead to treatment discontinuation and interruptions. An impaired renal function may affect the indication of neoadjuvant or adjuvant treatment in urothelial cancers, as well. Indeed, pts with a GFR $<60 \mathrm{~mL} /$ $\mathrm{min} / 1.73 \mathrm{~m}^{2}$ are generally excluded from pre and/or postoperative treatments due to high risks of toxicity [28, 29]. Many formulas to calculate the GFR have been developed and tested over the years in pts to determine the most effective and safest dose of chemotherapy as carboplatin or cisplatin, but none of these fully addressed the problems of complex pharmacokinetics and changing renal function [30].

Regarding nephrological pts, a reliable determination of renal function is crucial in many clinical situations, including the clinical evaluation of pts with renal insufficiency, the stage in CKD groups, the risk for disease progression, the indication for dialysis therapy, the screening living donors and the dose adjustment of toxic drugs, and so on [31]. Formulas are algorithms based on creatinine and or cystatin-c, age, and sex. However, both creatinine and cystatine-c are not perfect markers of renal function [5, 32]. Creatinine levels depend on muscle mass and protein intake and the levels of cystatin-c can be increased in obesity, subclinical inflammation and diabetes, independently of the level of renal function. This may explain, at least in part, the underestimation of renal function.

\section{Conclusion}

Direct measurement of GFR using a gold standard technique must be considered in selected pts before clinical decision. Assuming that performing mGFR for routine practice or in epidemiological studies could be not always feasible, the current findings emphasize the absolute need to determine an mGFR with gold standard method at least for those pts at eGFR lower than $60 \mathrm{~mL} / \mathrm{min} / 1.73 \mathrm{~m}^{2}$ who deserve surgical operations, anticoagulant therapies, nephrotoxic drugs, oncological medical therapies (included experimental protocols), and radiological contrast medium agents injections.

Despite the inaccuracy of eGFR formulas in comparison to gold standard methods is a well-known argument for nephrologists, this message needs to be spread in other clinical fields where the use eGFR prevails unchallenged.

\section{Limitation of the Study}

This study is characterized by some limitations due to the nature of the retrospective model design. In particular, the data available for the pts do not include comorbidities such as blood hypertension, diabetes, and obesity which could influence the renal health status. The second limitation is that in Center 2 no actual gold standard method is used to determine 
GFR making inappropriate to speculate on eGFR efficacy. The consequence of this limitation is the lack of a cohort where both mGFR techniques where used making impossible to merge the 2 cohorts.

\section{Statement of Ethics}

The study was conducted in accordance the World Medical Association Declaration of Helsinki. All pts had signed an informed consent agreeing to deliver their own anonymous information for future studies. The study was approved by local Ethical Committee.

\section{Disclosure Statement}

The authors have no conflicts of interest to declare.

\section{Funding Sources}

The funds for the printing fee of this manuscript are provided by an unrestricted grant from Recordati.

\section{Author Contributions}

The authors listed below have made substantial contribution to the intellectual content of the paper in the section described below. F.T., F.D.M., U.C., E.P., S.L.-L., F.D., and A.B.: conception and design. F.T., E.P., L.G., and G.D.C.: acquisition of data. F.D.M., F.T., E.P., and R.V.: analysis and interpretation of data. F.T., F.D.M., U.C., A.L., E.P., R.V., and M.G.: drafting of the manuscript. A.B., N.N.-M., and A.C.: critical revision of the manuscript for intellectual content. F.D.M., S.L.-L., and E.P.: analysis. A.S., E.P., and F.M.: supervision.

\section{References}

1 Luis-Lima S, Escamilla-Cabrera B, Negrin-Mena N, Estupinan S, Delgado-Mallen P, Marrero-Miranda D, et al Chronic kidney disease staging with cystatin C or creatinine-based formulas: flipping the coin. Nephrol Dial Transplant. 2019 Feb;34(2):287-94.

2 Jones GR. Estimating renal function for drug dosing decisions. Clin Biochem Rev. 2011 May;32(2):81-8.

3 Lindegaard MW, Aass N, Bue ES, Theodorsen L, Fosså SD. Glomerular filtration rate, 131I-hippuran clearance and estimated creatinine clearance in cancer patients. Br J Cancer. 1991 Aug; 64(2):401-5.

4 Hall YN, Himmelfarb J. The CKD Classification System in the Precision Medicine Era. Clin J Am Soc Nephrol. 2017 Feb;12(2):346-8.

5 Porrini E, Ruggenenti P, Luis-Lima S, Carrara F, Jiménez A, de Vries AP, et al. Estimated GFR: time for a critical appraisal. Nat Rev Nephrol. 2019 Mar;15(3):177-90.

6 National Kidney Foundation. K/DOQI clinical practice guidelines for chronic kidney disease: evaluation, classification, and stratification. Am J Kidney Dis. 2002 Feb;39(2 Suppl 1):S1-266.

7 Capitanio U, Montorsi F. Renal cancer. Lancet. 2016 Feb;387(10021):894-906.

8 Hornum M, Feldt-Rasmussen B. Drug Dosing and Estimated Renal Function - Any Step Forward from Effersoe? Nephron. 2017;136(4):268-72.

9 Hughes S, Szeki I, Nash MJ, Thachil J. Anticoagulation in chronic kidney disease patients-the practical aspects. Clin Kidney J. 2014 Oct;7(5):442-9.

10 Lentine KL, Kasiske BL, Levey AS, Adams PL, Alberú J, Bakr MA, et al. KDIGO Clinical Practice Guideline on the Evaluation and Care of Living Kidney Donors. Transplantation. 2017 Aug;101(8S Suppl 1):S1-109. 


\section{Kidney \\ Blood Pressure \\ Research}

\begin{tabular}{l|l}
\hline Kidney Blood Press Res 2020;45:166-179 \\
\hline DOI: 10.1159/000504649 & $\begin{array}{l}\text { @ 2020 The Author(s). Published by S. Karger AG, Basel } \\
\text { www.karger.com/kbr }\end{array}$ \\
\hline
\end{tabular}

Trevisani et al.: New Challenges in Renal Function Assessment

11 Luis-Lima S, Gaspari F, Porrini E, García-González M, Batista N, Bosa-Ojeda F, et al. Measurement of glomerular filtration rate: internal and external validations of the iohexol plasma clearance technique by HPLC. Clin Chim Acta. 2014 Mar;430:84-5.

12 Luis-Lima S, Gaspari F, Negrín-Mena N, Carrara F, Díaz-Martín L, Jiménez-Sosa A, et al. Iohexol plasma clearance simplified by dried blood spot testing. Nephrol Dial Transplant. 2018 Sep;33(9):1597-603.

13 Assadi M, Eftekhari M, Hozhabrosadati M, Saghari M, Ebrahimi A, Nabipour I, et al. Comparison of methods for determination of glomerular filtration rate: low and high-dose Tc-99m-DTPA renography, predicted creatinine clearance method, and plasma sample method. Int Urol Nephrol. 2008;40(4):1059-65.

14 Levey AS, Stevens LA, Schmid CH, Zhang YL, Castro AF 3rd, Feldman HI, et al.; CKD-EPI (Chronic Kidney Disease Epidemiology Collaboration). A new equation to estimate glomerular filtration rate. Ann Intern Med. 2009 May;150(9):604-12.

15 Levey AS, Bosch JP, Lewis JB, Greene T, Rogers N, Roth D; Modification of Diet in Renal Disease Study Group. A more accurate method to estimate glomerular filtration rate from serum creatinine: a new prediction equation. Ann Intern Med. 1999 Mar;130(6):461-70.

16 Pottel H, Delanaye P, Schaeffner E, Dubourg L, Eriksen BO, Melsom T, et al. Estimating glomerular filtration rate for the full age spectrum from serum creatinine and cystatin C. Nephrol Dial Transplant. 2017 Mar;32(3): 497-507.

17 Rule AD, Larson TS, Bergstralh EJ, Slezak JM, Jacobsen SJ, Cosio FG. Using serum creatinine to estimate glomerular filtration rate: accuracy in good health and in chronic kidney disease. Ann Intern Med. 2004 Dec; 141(12):929-37.

18 Le Bricon T, Thervet E, Froissart M, Benlakehal M, Bousquet B, Legendre C, et al. Plasma cystatin C is superior to 24-h creatinine clearance and plasma creatinine for estimation of glomerular filtration rate 3 months after kidney transplantation. Clin Chem. 2000 Aug;46(8 Pt 1):1206-7.

19 Rule AD, Bergstralh EJ, Slezak JM, Bergert J, Larson TS. Glomerular filtration rate estimated by cystatin C among different clinical presentations. Kidney Int. 2006 Jan;69(2):399-405.

20 Inker LA, Schmid CH, Tighiouart H, Eckfeldt JH, Feldman HI, Greene T, et al.; CKD-EPI Investigators. Estimating glomerular filtration rate from serum creatinine and cystatin C. N Engl J Med. 2012 Jul;367(1):20-9.

21 Stevens LA, Zhang Y, Schmid CH. Evaluating the performance of equations for estimating glomerular filtration rate. J Nephrol. 2008 Nov-Dec;21(6):797-807.

22 Lin LI. Total deviation index for measuring individual agreement with applications in laboratory performance and bioequivalence. Stat Med. 2000 Jan;19(2):255-70.

23 Capitanio U, Terrone C, Antonelli A, Minervini A, Volpe A, Furlan M, et al. Nephron-sparing techniques independently decrease the risk of cardiovascular events relative to radical nephrectomy in patients with a T1a-T1b renal mass and normal preoperative renal function. Eur Urol. 2015 Apr;67(4):683-9.

24 Sheir KZ, Gad HM. Prospective study of the effects of shock wave lithotripsy on renal function: role of postshock wave lithotripsy obstruction. Urology. 2003 Jun;61(6):1102-6.

25 Chen J, Wang XT, Luo PH, He QJ. Effects of unidentified renal insufficiency on the safety and efficacy of chemotherapy for metastatic colorectal cancer patients: a prospective, observational study. Support Care Cancer. 2015 Apr;23(4):1043-8.

26 Launay-Vacher V, Oudard S, Janus N, Gligorov J, Pourrat X, Rixe O, et al.; Renal Insufficiency and Cancer Medications (IRMA) Study Group. Prevalence of Renal Insufficiency in cancer patients and implications for anticancer drug management: the renal insufficiency and anticancer medications (IRMA) study. Cancer. 2007 Sep; 110(6):1376-84.

27 Cathomas R, Klingbiel D, Geldart TR, Mead GM, Ellis S, Wheater M, et al. Relevant risk of carboplatin underdosing in cancer patients with normal renal function using estimated GFR: lessons from a stage I seminoma cohort. Ann Oncol. 2014 Aug;25(8):1591-7.

28 Dash A, Galsky MD, Vickers AJ, Serio AM, Koppie TM, Dalbagni G, et al. Impact of renal impairment on eligibility for adjuvant cisplatin-based chemotherapy in patients with urothelial carcinoma of the bladder. Cancer. 2006 Aug;107(3):506-13.

29 Thompson RH, Boorjian SA, Kim SP, Cheville JC, Thapa P, Tarrel R, et al. Eligibility for neoadjuvant/adjuvant cisplatin-based chemotherapy among radical cystectomy patients. BJU Int. 2014 May;113 5b:E17-21.

30 Holweger K, Lipp HP, Dietz K, Hartmann JT, Bokemeyer C. Novel algorithm for more accurate calculation of renal function in adults with cancer. Ann Pharmacother. 2008 Dec;42(12):1749-57.

31 Porrini E, Warnock DG. Evaluation of Renal Function and Renal Risk in the Twenty-First Century. Nephron. 2017;136(4):261-2.

32 Luis-Lima S, Porrini E. An Overview of Errors and Flaws of Estimated GFR versus True GFR in Patients with Diabetes Mellitus. Nephron. 2017;136(4):287-91. 